\title{
DESIGN OF AN ENHANCED 1 GeV ELECTRON NEUTRINO BEAM
}

\author{
R.A. ONG ${ }^{*}$, L. SULAK and R. WATTS \\ Randall Laboratory, University of Michigan, Ann Arbor, MI 48100, USA
}

Received 7 November 1984

\begin{abstract}
An enhanced $\nu_{\mathrm{e}}$ beam would be useful for experiments that have been proposed to investigate the time evolution of a $\nu_{\mathrm{e}}$ beam or to test the universality of $\nu_{\mathrm{e}}$ and $\nu_{\mu}$ interactions. We have performed detailed calculations to maximize the flux of electron neutrinos through a detector while minimizing the more copious muon neutrinos produced at $30 \mathrm{GeV}$ proton accelerators. The $\nu_{\mathrm{e}}$ beam is formed from the semi-leptonic decay modes of the neutral kaon. Muon neutrinos generated by decays of charged pions and kaons are suppressed by a dipole sweeping magnet. The $\nu_{\mathrm{e}} / \nu_{\mu}$ ratio is enhanced from its usual value of $\sim 1 / 1000$ to a value of $\sim 1 / 2$, albeit at a low flux. We find with this design a typical flux of $1.5 \times 10^{7} \nu_{\mathrm{e}} / \mathrm{m}^{2} \mathrm{~s}$. We find that a high magnetic field ( $\geq 40 \mathrm{kG}$ ) is essential to achieve this $\nu_{\mathrm{c}} / \nu_{\mu}$ enhancement. Although the use of collimators and/or plugs inside the magnet reduces the $\nu_{\mu}$ flux, the $\nu_{\mathrm{e}}$ flux is also diminished so that there is little beneficial effect on the $\nu_{\mathrm{e}} / \nu_{\mu}$ ratio. Magnetic focusing horns and quadrupole beams do not enhance the $\nu_{e} / \nu_{\mu}$ ratio. The accuracy of the energy dependence of the calculation, as well as the absolute normalization of the fluxes, is determined by a subsidiary calculation of the $\nu_{\mu}$ yield from the magnetic horn focused beam at Brookhaven National Laboratory. This calculation is the first to our knowledge to agree well with the $\nu_{\mu}$ yield as measured in the BNL seven foot bubble chamber.
\end{abstract}

\section{Introduction}

Currently there is a good deal of interest in the possibility of neutrino oscillations and non-zero neutrino masses. This interest stems from the crucial role the neutrino plays in theories which unify the strong and electroweak interactions. Several experiments [1] have studied the time evolution of an accelerator $\nu_{\mu}$ beam with reasonably high sensitivity. Because of low $\nu_{\mathrm{e}}$ production however (typically one $\nu_{\mathrm{e}}$ for every $1000 \nu_{\mu}$ ), only a few experiments have obtained information regarding the time evolution of a $\nu_{e}$ beam. This lack of information on accelerator $\nu_{\mathrm{e}}$ 's is unfortunate since the only published indications of possible oscillations and non-zero neutrino mass occur in the electron neutrino sector. The Savannah River and Bugey reactor experiments have reported the possible disappearance of $\bar{\nu}_{e}$ 's as detected downstream from the reactor source [2]. The CHARM collaboration beam dump experiment at the CERN SPS reports an anomalous prompt $\nu_{\mathrm{e}}: \nu_{\mu}$ ratio of $1: 2$ as opposed to the $1: 1$ ratio expected from the decay of charmed parents [3]. One interpretation of this discrepancy [4] is that the electron neutrinos oscillate into other sectors while travelling from the source to the detectors. The ITEP group has improved their analysis of the end point energy in tritium beta decay and claims

\footnotetext{
* Present address: Stanford Linear Accelerator Center, Stanford CA, 94305, USA.
}

that recent results continue to support a $\nu_{\mathrm{e}}$ mass greater than $20 \mathrm{eV}$ [5]. Finally, the apparent deficiency of the solar $\nu_{\mathrm{e}}$ 's as seen in the Homestake Gold Mine experiment experiment could be interpreted as a result of neutrino oscillations [6].

Phenomenologically, neutrino oscillations are characterized in terms of the evolution in proper time of a massive neutrino. In convenient units this is $L / p$ where $L$ is the neutrino flight path and $p$ is the neutrino momentum. Typically, reactor experiments have explored the region of $L / p$ around $10 \mathrm{~m} / 5 \mathrm{MeV}=2$ $\mathrm{km} / \mathrm{GeV}$, while the CERN SPS experiments have looked at the region around $900 \mathrm{~m} / 25 \mathrm{GeV}=0.04 \mathrm{~km} / \mathrm{GeV}$ [7]. These regions in $L / p$ correspond to possible mass differences between neutrino eigenstates of 1 and $50 \mathrm{eV}$ respectively. In examining these regions, however, both :eactor and accelerator based experiments have been hampered by systematic problems. For the reactors, problems center around the scarcity of $\nu_{e}$ 's and the knowledge of the energy dependence of the neutrino flux; for the beam dump experiments, problems involve the unknown production mechanism of the $\nu_{e}$ 's and the low $\nu_{\mathrm{e}} / \nu_{\mu}$ ratio.

Thus it appears that a neutrino oscillation experiment capable of exploring a large range of $L / p$ and optimized to search for oscillatory behavior of the $\nu_{\mathrm{e}}$ sector is desirable. Certainly, the experiment should cover the region of phase space delineated by the recent indications of possible effects $\left(L / p=10^{-2}\right.$ to 1 $\mathrm{km} / \mathrm{GeV}$ ), and be sensitive to oscillation effects at the 
$5 \%$ level or better, the level expected if neutrino mixing angles are comparable to quark mixing angles between the first two generations. To date there have been no copious accelerator $\nu_{e}$ sources available for such a study, and certainly no $\nu_{\mathrm{e}}$ sources with low contamination of $\nu_{\mu}$ 's.

For a $\nu_{\mathrm{e}}$ source, the $30 \mathrm{GeV}$ proton accelerators have clear advantages over alternative neutrino sources [8]. Since they accelerate the highest flux of protons above kaon production threshold [9], the $30 \mathrm{GeV}$ proton machines have the potential for producing the largest number of $\nu_{\mathrm{e}}$ 's. Furthermore, the neutrino energy range $(0.5-4 \mathrm{GeV})$ and accessible detector distances $(40 \mathrm{~m}-1.5$ $\mathrm{km}$ ) naturally yield the desired $L / p$ range. Although high fluxes of low energy $\nu_{\mathrm{e}}$ 's from muon decay are available at pion factories, they are limited in several ways. The $53 \mathrm{MeV}$ maximum neutrino energy places an upper limit on the sensitivity to neutrino mass differences for typical detector distances. A lower limit at LAMPF comes from the $6 \%$ duty cycle which leads to an unavoidable cosmic ray background at large flight paths (where the event rate is necessarily low). Furthermore, the $\nu_{\mathrm{e}}$ 's from muons decaying at rest are produced isotropically. Thus one lacks the intensification gained by the Lorentz focusing inherent in neutrino beams produced by decay in flight. Going to higher energies at Fermilab or the SPS at CERN turns out not to be advantageous. Neutrinos produced at these facilities are too high in energy to achieve adequate sensitivity to small neutrino mass differences. In addition, neutrino production processes above charm threshold are not well understood thus preventing unambiguous interpretations of deviations from nominal neutrino event rates.

The most favorable conditions for production of a $\nu_{e}$ beam to be used for oscillation studies therefore appear to be at the $30 \mathrm{GeV}$ proton accelerators, the Brookhaven AGS or the CERN PS. Such studies have been suggested $[10,11]$. To determine the feasibility of the proposed experiments, an understanding of the $\nu_{\mathrm{e}}$ flux, both in energy dependence and in absolute magnitude, is needed as a function of distance from the production region. In addition, suppression of $\nu_{\mu}$ production must be optimized and the remaining $\nu_{\mu}$ background evaluated. To address these issues, we have performed a detailed computer simulation, incorporating all known aspects of the production and propagation of a neutrino beam.

We first outline the structure of the simulation. We then test it by comparing our generated $\nu_{\mu}$ spectrum with an experimentally determined one. We conclude with an explicit example of a possible design for a $\nu_{\mathrm{e}}$ beam at BNL, the prediction of the $\nu_{\mathrm{e}}$ energy spectrum, and an evaluation of the accompanying $\nu_{\mu}$ background.

\section{General description of the $v_{e}$ beam}

The salient characteristics of possible $\nu_{\mathrm{e}}$ beams at BNL and at CERN are quite similar. For concreteness, we pursue the parameters of the suggestions initially made by the authors [10]. Fig. 1 shows the first $100 \mathrm{~m}$ of the beamline. Incoming $28 \mathrm{GeV}$ protons from the AGS strike a pencil target producing a secondary beam composed primarily of pions and kaons. A list of the simulated secondary particles, along with their decay products and branching ratios, is given in table 1 . Other particles and decay modes are assumed to be insignificant. After production, most charged particles are swept out of the beam by the strong dipole sweeping magnet surrounding the target. The remaining particles are then allowed to decay while they travel downstream to the iron muon shield. The shield is assumed to absorb everything but the neutrinos. They pass into the detector and are counted. The neutrino detector consists of several massive water Cherenkov modules judiciously spaced along the neutrino beam line. Fig. 1 shows the location of the first detector module $90 \mathrm{~m}$ from the target. Further detector modules are located at 250, 500 and $1500 \mathrm{~m}$ from the target. For the purposes of this study we will determine the neutrino flux into a detector of three meters in diameter placed directly behind the iron shield.

The electron neutrino beam is generated primarily from the semi-leptonic decays of $\mathrm{K}_{\mathrm{L}}^{0}$ 's. In principle, one would also like to use $\mathrm{K}^{+}$semi-leptonic decays, but there appears to be no way to suppress the dominant background of $\nu_{\mu}$ 's from $\pi^{+}$decay without also suppressing the $\nu_{e}$ 's from $\mathrm{K}^{+}$decay. The major source of background in the detector is due to $\nu_{\mu}$ events which masquerade as $\nu_{\mathrm{e}}$ events. To cut down the number of $\nu_{\mu}$ 's entering the detector and thus increase the signal to noise ratio, a magnet is used to sweep out the charged pions and kaons that give rise to the majority of $\nu_{\mu}$ 's. The residual $v_{\mu}$ 's which arrive at the detector result from secondaries that decay before being sufficiently bent out of the beam and from the products of $\mathrm{K}_{\mathrm{L}}^{0}$ and $\Lambda^{0}$ decay.

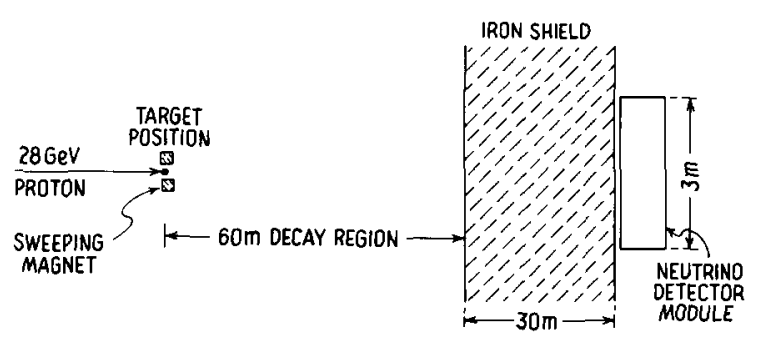

Fig. 1. Layout of first $100 \mathrm{~m}$ of BNL $\nu_{e}$ beam line. 
Table 1

Decay products and branching ratios

\begin{tabular}{lll}
\hline Secondary & Decay mode & Branching ratio \\
\hline$\pi^{+}$ & $\nu_{\mu} \mu^{+}$ & 1.00 \\
$\pi^{-}$ & $\bar{\nu}_{\mu} \mu^{-}$ & 1.00 \\
$\mathrm{~K}^{+}$ & $\nu_{\mu} \mu^{+}$ & 0.64 \\
& $\pi^{+} \pi^{0}$ & 0.21 \\
& $\pi^{+} \pi^{+} \pi^{-}$ & 0.056 \\
$\mathrm{~K}^{-}$ & $\bar{\nu}_{\mu} \mu^{-}$ & 0.64 \\
& $\pi^{-} \pi^{0}$ & 0.21 \\
& $\pi^{+} \pi^{-} \pi^{-}$ & 0.056 \\
$\mathrm{~K}_{\mathrm{S}}^{0}$ & $\pi^{+} \pi^{-}$ & 0.69 \\
$\mathrm{~K}_{\mathbf{L}}^{0}$ & $\pi^{+} \mathrm{e}^{-} \bar{\nu}_{\mathrm{e}}$ & 0.19 \\
& $\pi^{-} \mathrm{e}^{+} \nu_{\mathrm{e}}$ & 0.19 \\
& $\pi^{+} \mu^{-} \bar{\nu}_{\mu}$ & 0.14 \\
& $\pi^{-} \mu^{+} \nu_{\mu}$ & 0.14 \\
$\mu^{+}$ & $\pi^{+} \pi^{-} \pi^{0}$ & 0.12 \\
$\mu^{-}$ & $\mathrm{e}^{+} \bar{\nu}_{\mu} \nu_{\mathrm{e}}$ & 0.19 \\
$\Lambda^{0}$ & $\mathrm{e}^{-} \nu_{\mu} \bar{\nu}_{\mathrm{e}}$ & 0.99 \\
\hline
\end{tabular}

\section{Details of the $v_{\mathrm{e}}$ and $v_{\mu}$ flux simulation}

The simulation was written in FORTRAN for use on a DEC VAX-780 computer. The significant parameters used in the simulation are listed in tables 2 and 3. Described below are the important features of the simulation.

The incident proton beam is modeled as a normally incident, radially symmetric Gaussian distribution with

Table 2

Parameters of BNL $\nu_{\mu}$ beam line with focusing horn

\begin{tabular}{ll}
\hline Proton momentum & $27.8 \mathrm{GeV} / \mathrm{c}$ \\
$\begin{array}{l}\text { Target: } \\
\text { length }\end{array}$ & $52.5 \mathrm{~cm}$ \\
radius & $0.3175 \mathrm{~cm}$ \\
material & sapphire $\left(\mathrm{Al}_{2} \mathrm{O}_{3}\right)^{\text {a) }}$ \\
density & $3.987 \mathrm{~g} / \mathrm{cm}^{3}$
\end{tabular}

a) Sapphire target is modeled as beryllium since the Eichten data shows little difference between $\mathrm{Al}$ and $\mathrm{Be}$. a spatial $\sigma_{r}=1.4 \mathrm{~mm}$, and a Gaussian angular distribution with $\sigma_{\theta}=2.5 \mathrm{mrad}[12]$. The beam is exponentially absorbed in the target. The interaction vertex is randomly picked according to these constraints. Typically, $20 \%$ of the incident protons do not interact with the target and are ignored by the program. The target is assumed to be beryllium, with an absorption length of $36.7 \mathrm{~cm}$. Beryllium is chosen because it produces the highest kaon yield per target interaction [13]. Subsequent interactions of each proton with the target are ignored.

Secondary pions and kaons resulting from $p-B e$ collisions are generated by using the production spectra measured by Eichten et al. at CERN [13]. To obtain an analytic expression for the production spectra, a modified version of the 1967 Sanford-Wang parametrization is used as an interpolating tool [14]:

$$
\begin{aligned}
& \frac{\mathrm{d}^{2} N}{\mathrm{~d} \Omega \mathrm{d} P} \mid \begin{array}{l}
(\# / \mathrm{SrGeV} / c)=C_{1} P^{C_{2}}\left(1-\frac{P}{P_{\mathrm{i}}}\right) \\
\text { Sanford-Wang }
\end{array} \\
& \quad \times \exp \left[\frac{-C_{3} P^{C_{4}}}{P^{C_{5}}}-C_{6} \theta\left(P-C_{7} P_{\mathrm{i}} \cos ^{C_{8} \theta}\right)\right] .
\end{aligned}
$$

where: $P=$ momentum of secondary particle,

$\theta=$ the production angle of the secondary,

$P_{i}=$ momentum of incident proton, and

$C_{1}-C_{8}=$ fitting coefficients listed in table 4 .

This parametrization is modified so that the results match the experimental data of Eichten et al. at 24 $\mathrm{GeV} / c$. The modification for the $\pi^{+}, \pi^{-}$, and $\mathrm{K}^{+}$

Table 3

\begin{tabular}{|c|c|}
\hline Proton momentum & $28 \mathrm{GeV} / c$ \\
\hline $\begin{array}{l}\text { Target: } \\
\text { length } \\
\text { radius } \\
\text { material } \\
\text { density }\end{array}$ & $\begin{array}{l}75 \mathrm{~cm} \\
0.25 \mathrm{~cm} \\
\mathrm{Be} \\
1.848 \mathrm{~g} / \mathrm{cm}^{3}\end{array}$ \\
\hline $\begin{array}{l}\text { Magnet: BNL } 18 \text { D } 72 \\
\text { length } \\
\text { gap } \\
\text { field }\end{array}$ & $\begin{array}{l}72^{\prime \prime} \\
6^{\prime \prime} \times 19^{\prime \prime} \\
22 \mathrm{kG}\end{array}$ \\
\hline $\begin{array}{l}\text { Decay tunnel: } \\
\text { length } \\
\text { radius }\end{array}$ & $\begin{array}{l}60 \mathrm{~m} \\
2 \mathrm{~m}\end{array}$ \\
\hline $\begin{array}{l}\text { Iron shield: } \\
\text { length }\end{array}$ & $30 \mathrm{~m}$ \\
\hline $\begin{array}{l}\text { Model detector: } \\
\text { radius } \\
\text { location } \\
\text { fiducial mass }\left(\mathrm{H}_{2} \mathrm{O}\right)\end{array}$ & $\begin{array}{l}1.5 \mathrm{~m} \\
\text { following iron shield } \\
1 \mathrm{kt}\end{array}$ \\
\hline
\end{tabular}

Parameters of BNL $\nu_{e}$ enriched beam line 
Table 4

Coefficients for modified Sanford-Wang parametrization

\begin{tabular}{lllllllll}
\hline Secondary & $C_{1}$ & $C_{2}$ & $C_{3}$ & $C_{4}$ & $C_{5}$ & $C_{6}$ & $C_{7}$ & $C_{8}$ \\
\hline$\pi^{+}$ & 1.092 & 0.6458 & 4.046 & 1.625 & 1.656 & 5.029 & 0.1722 & 82.65 \\
$\pi^{-}$ & 0.821 & 0.5271 & 3.956 & 1.731 & 1.617 & 4.735 & 0.1984 & 88.75 \\
$\mathrm{~K}^{+}$ & 0.059 & 0.6916 & 3.744 & 4.520 & 4.190 & 4.928 & 0.1922 & 50.28 \\
\hline
\end{tabular}

secondaries is

$$
\begin{aligned}
& \frac{\mathrm{d}^{2} N}{\mathrm{~d} \Omega \mathrm{d} P}=\left.\frac{\mathrm{d}^{2} N}{\mathrm{~d} \Omega \mathrm{d} P}\right|_{\text {Sanford-Wang }} \\
& \quad \times F(P)(1.0+C P)\left[1+A\left(\theta-\theta_{0}\right)+B\left(\theta-\theta_{0}\right)^{2}\right],
\end{aligned}
$$

where the coefficients $A, B$ and $C$ are given in table 5, and the function $F(P)$ is given in table 6. Although the modified production spectra fit the Eichten data well, we fix the fit at $\theta_{0}=17 \mathrm{mrad}$, the average acceptance of a typical detector. The $\mathrm{K}^{-}$production spectra are derived from the $\mathrm{K}^{+}$spectra by the following empirical fit:

$$
\frac{\mathrm{d}^{2} N}{\mathrm{~d} \Omega \mathrm{d} P}\left(\mathrm{~K}^{-}\right)=\left\{\begin{array}{c}
\frac{\mathrm{d}^{2} N}{\mathrm{~d} \Omega \mathrm{d} P}\left(\mathrm{~K}^{+}\right) \frac{F(P)}{2.7}, \\
\text { for } P<4 \mathrm{GeV} / c, \\
\frac{\mathrm{d}^{2} N}{\mathrm{~d} \Omega \mathrm{d} p}\left(\mathrm{~K}^{+}\right) \frac{F(P)}{\left[2.7+0.134(P-4.0)^{2}\right]}, \\
\text { for } P \geq 4 \mathrm{GeV} / c .
\end{array}\right.
$$

Figs. 2 and 3 compare generated $\pi^{+}$and $\mathrm{K}^{+}$spectra against Eichten's data for secondary momenta of 5 and $8 \mathrm{GeV} / c$ and for production angles from 0 to $100 \mathrm{mrad}$.

\begin{tabular}{|c|c|c|c|}
\hline Secondary & $A$ & $B$ & $C$ \\
\hline$\overline{\pi^{+}}$ & $-1.0 \times 10^{-3}$ & $1.0 \times 10^{-6}$ & 0.45 \\
\hline$\pi^{-}$ & $-5.0 \times 10^{-3}$ & $1.5 \times 10^{-5}$ & 0.0 \\
\hline $\mathrm{K}^{+}$ & $2.0 \times 10^{-4}$ & $-2.3 \times 10^{-6}$ & 4.0 \\
\hline
\end{tabular}

Table 5

Coefficients for modified Sanford-Wang parametrization

Table 6

Coefficients for modified Sanford-Wang parametrization

\begin{tabular}{lll}
\hline Secondary & $F(p)$ & \\
\hline$\pi^{+}$ & $0.1424 p^{2} \mathrm{e}^{-0.1809 p}$ & $p>4 \mathrm{GeV} / c$ \\
& $0.5985 p$ & $p \leq 4 \mathrm{GeV} / c$ \\
$\pi^{-}$ & $0.4443 p \mathrm{e}^{-0.0713 p}$ & $p>4 \mathrm{GeV} / c$ \\
& $0.7044 p$ & $p \leq 4 \mathrm{GeV} / c$ \\
$\mathrm{~K}^{+}$ & 1.273 & $p>4 \mathrm{GeV} / c$ \\
& $0.0155 p \mathrm{e}^{-0.0713 p}$ & $p \leq 4 \mathrm{GeV} / c$ \\
$\mathrm{~K}^{-}$ & $0.2355 p$ & $p<4 \mathrm{GeV} / c$ \\
\hline
\end{tabular}



Fig. 2. Angular production spectra comparison for $\pi^{+}$at 5 $\mathrm{GeV} / c$ and at $8 \mathrm{GeV} / c$. Solid line is interpolated spectrum described in text while points with error bars are data of Eichten et al.

Comparisons at other momenta and for $\pi^{-}$and $\mathrm{K}^{-}$ secondaries show similar good agreement to the Eichten data. The bulk of the detected neutrino signal comes from these regions in the spectra. Note that the parameterization gives results which do not diverge at low momenta and at zero angle where no data exists. To obtain the $\mathrm{K}_{\mathrm{L}}^{0}$ spectra, isospin invariance is invoked and the average of the $\mathrm{K}^{+}$and $\mathrm{K}^{-}$spectra is taken at the desired momentum and angle. For each momentum and polar angle generated, a random azimuthal angle is chosen.

Secondaries produced in the target are propagated through the target in $1 \mathrm{~cm}$ increments. At each step the particle suffers $\mathrm{d} E / \mathrm{d} x$ ionization loss and bending in the magnetic field. Since the typical multiple Coulomb scattering angle is much less than the production angle, it is ignored. Secondaries that are found to have been scattered inelastically are assumed to be completely removed from the system, along with any tertiary particles they may create. Inelastic scattering supresses the

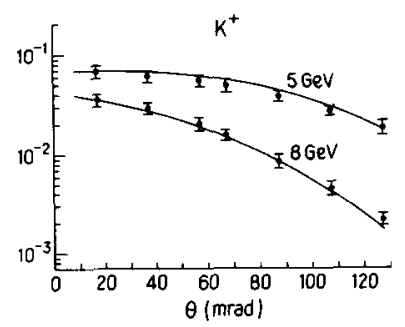

Fig. 3. Angular production spectra comparison for $\mathrm{K}^{+}$at $\mathrm{s}$ $\mathrm{GeV} / c$ and at $8 \mathrm{GeV} / c$. 
$\nu_{\mathrm{e}}$ flux from $\mathrm{K}_{\mathrm{L}}^{0}$ decays, since the majority of the $\nu_{\mathrm{e}}$ 's that arrive at the detector come from $\mathrm{K}_{\mathrm{L}}^{0}$ 's that travel the entire length of the target at low production angles $(<30 \mathrm{mrad})$.

In addition to the target, we consider absorption of particles in the magnet system, the decay tunnel and the iron shield. Because the magnetic field region depends on the experimental configuration, a discussion of absorption by each configuration is presented below. Absorption at the decay tunnel walls is assumed to be total, i.e. the particle and all potential products of the absorptive interaction are removed from consideration. Likewise, all particles other than neutrinos are totally absorbed by the iron shield.

All decays are assumed to be isotropic in the center of mass except for the semi-leptonic neutral kaon decays and the $\mu \rightarrow \nu_{\mu} \nu_{\mathrm{e}} \mathrm{e}$ decay. The kaon decays are calculated according to the distributions given by the V-A theory and observed Dalitz plot data [15]. The production of the two neutrinos from muon decay assumes a $\mathbf{V}-\mathbf{A}$ interaction.

\section{Comparison of simulation with existing $\nu_{\mu}$ spectra}

To check the simulation described above, we test its predictions against known data. In particular, to determine the absolute normalization of the flux generated by the simulation, the parameters of the program were modified to simulate the muon neutrino data taken by Murtagh et al., in the Brookhaven seven foot bubble chamber [16]. The physical parameters of this beam line are given in table 2 [17]. The focusing system of the Brookhaven neutrino beam line is a double magnetic horn. It is a set of concentric conductors that focus charged pions and kaons, and hence increase the $\nu_{\mu}\left(\bar{\nu}_{\mu}\right)$ flux. The complex structure of the conductors is modeled as a set of cylinders and cones enclosing a magnetic field falling off as $1 / r$. Because of the shape of the horn, an analytic solution for particle trajectories is not attempted. Instead, the particles are stepped through the system in small increments.

Fig. 4 shows the transfer curves for 5 and $7 \mathrm{GeV}$ $\pi^{+}$'s created on axis approximately halfway down the length of the target. The cusps in the figures are the result of discontinuities in the magnet geometry. To obtain these curves, the particles are propagated through the horn in $1 \mathrm{~mm}$ steps. However, this procedure requires a large amount of computing time. A coarser grid is used to generate many events: the particle is moved in steps of $1 \mathrm{~cm}$ for a radial distance less than $6 \mathrm{~cm}, 5 \mathrm{~cm}$ for a radial distance less than $18 \mathrm{~cm}$, and $10 \mathrm{~cm}$ otherwise. In this procedure, the field inhomogeneity across a single step is always less than $15 \%$ and the transmission curves are in good agreement with fig. 4 . As can be seen

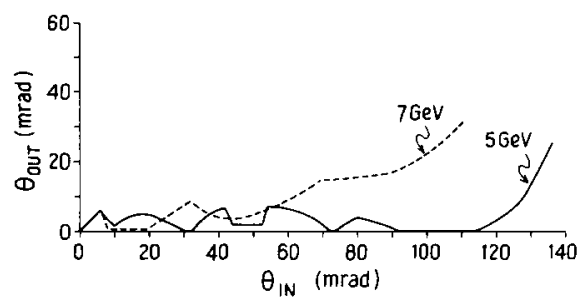

Fig. 4. Transfer curves for BNL horn magnet system. Curves are for 5 and $7 \mathrm{GeV} / c \pi^{+}$'s created $25 \mathrm{~cm}$ into the target.

from this figure, the magnet system is excellent at focusing particles up to $100 \mathrm{mrad}$, depending on the momentum. Fig. 5 shows the trajectories of $5 \mathrm{GeV} \pi^{+}$'s created at several positions and production angles in the target. As an example, a $\pi^{+}$created $25 \mathrm{~cm}$ into the target with an angle of $50 \mathrm{mrad}$ is almost perfectly focused at the end of $2 \mathrm{~m}$. In fact, all $\pi^{+}$'s created with an angle less than $137 \mathrm{mrad}$ are focused into the 34 mrad acceptance of the decay tunnel. With the magnetic horn set to focus positively charged particles and defocus negative ones, the typical focusing factor for $\pi$ 's is 23.9 and the typical defocusing factor is 5.0. These factors are calculated for those neutrinos that make it into a $1.1 \mathrm{~m}$ diameter detector located $90 \mathrm{~m}$ downstream from the target, a reasonable representation of the BNL seven foot chamber.

Absorption of particles by the horn system is taken into account as the particles are transported through the magnets. The inner and outer conductors of each horn are taken to be $1 / 8$ inch thick aluminum and the first horn has a $7 / 8$ inch thick aluminum end plate. Absorption is assumed to be total. In addition, the target is cooled by a water jacket between it and the inner

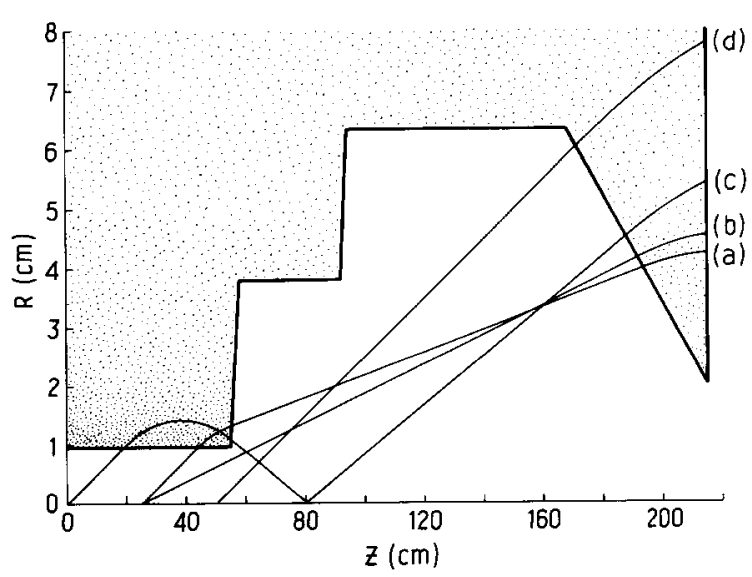

Fig. 5. Trajectories of a $5 \mathrm{GeV} \pi^{+}$through the first BNL horn magnet. (a) $\theta=50 \mathrm{mrad}$ created at $z=25 \mathrm{~cm}$. (b) $\theta=25 \mathrm{mrad}$, $z=25 \mathrm{~cm}$. (c) $\theta=40 \mathrm{mrad}, z=0 \mathrm{~cm}$. (d) $\theta=75 \mathrm{mrad}, z=25$ $\mathrm{cm}$. 
conductor of the first horn segment. The effects of absorption by this water are not included in the simulation. Similarly, absorption by additional flanges, bolts, etc. are ignored.

Fig. 6 displays the muon neutrino flux through the BNL seven foot chamber for neutrinos from $\pi^{+}$and $\mathrm{K}^{+}$secondaries as calculated by the simulation. Error bars in this figure and in the following ones are statistical only. The sum of these fluxes is normalized with the observed $\nu_{\mu}$ flux in fig. 7. The observed flux is obtained by identifying quasi-elastic neutrino events in the fiducial volume of the bubble chamber and assuming that the neutrino flux is uniform across the diameter of the chamber. Also displayed in this figure is the flux as calculated by Tso [18]. Absolute neutrino flux calculations have traditionally been up to an order of magnitude higher than observed fluxes due to the inability to account for targeting efficiency, beam monitoring etc. The results of our simulation are a factor of 3.6 higher than the BNL data. This normalization factor is included in all subsequent calculations. The simulation shape and the BNL data agree well in all regions, with excellent agreement from 0.5 to $2.0 \mathrm{GeV}$. Our calculations fit the data well at high energy, where the Tso spectrum is a factor of two too high. Note that the contributions to the $\nu_{\mu}$ flux from the decay of $\mathrm{K}^{0}$ secondaries and from the decay of tertiary muons are negligible.

There are other predictions of the BNL muon neu-



Fig. 6. Unnormalized components of the simulated $\nu_{\mu}$ BNL beam. Error bars in this and all subsequent figures are statistical only.

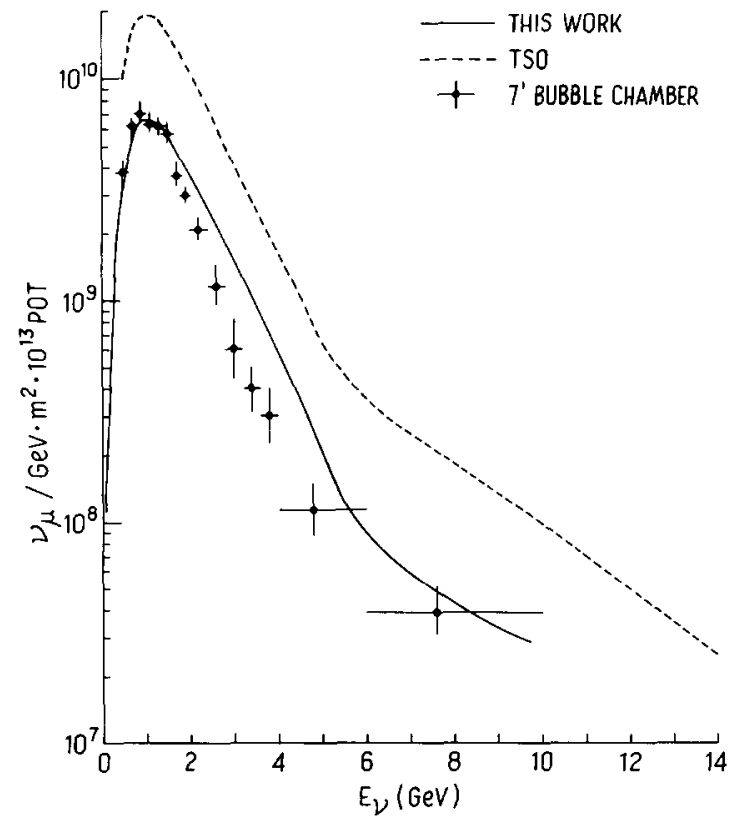

Fig. 7. Plot of BNL $\nu_{\mu}$ fluxes.

trino flux [19]. Such predictions either peak at a higher energy than does the data, have a larger flux at higher energy, or have both problems. To our knowledge, the simulation of this paper is the best fit to date of the Brookhaven $\nu_{\mu}$ flux.

\section{Design of an enriched $v_{e}$ beam}

The main goal of the $\nu_{\mathrm{e}}$ beam design is to minimize the number of $\nu_{\mu}$ 's entering the detector by sweeping charged pions and kaons out of the beam before they decay. In this way, the $\nu_{\mathrm{e}} / \nu_{\mu}$ ratio can be increased by about two orders of magnitude over a configuration without a sweeping magnet. The final design parameters are listed in table 3 . The length of the target is adjusted to maximize $\nu_{\mathrm{e}}$ 's from kaon decay at the first proposed detector position. The entire target is placed inside a magnetic field to sweep as many charged secondaries out of the beam as possible.

We modeled a $18 \mathrm{D} 72$ dipole magnet with a field strength of $22 \mathrm{kG}$ [20]. Because of the large amount of iron surrounding the gap of the magnet (at least one absorption length in every direction) absorption of secondaries by the magnet is assumed to be total.

Because collimation of secondary beams had been used in previous neutral particle experiments, collimation was attempted in this design. However, as figs. 2 and 3 show, pions are generally produced with less transverse momentum that kaons. Collimation stops more kaons than pions, decreasing the $\nu_{e} / \nu_{\mu}$ ratio. 
In another attempt at increasing the $\nu_{\mathrm{e}} / \nu_{\mu}$ ratio by selectively absorbing pions over kaons, a tungsten plug was inserted directly after the target. This also decreased the ratio. Only the kaons produced very forwardly yield neutrinos at the detector. The plug in the magnet then stopped these kaons before they decayed. Few pions were stopped since the large majority of them had already been swept out of the plug's path by the magnetic field.

The detector modeled consists of several modules of Cherenkov ring-imaging water calorimeters [10]. Each module of the detector consists of a fiducial volume of 1 kt of water viewed by photomultiplier tubes. The first detector is located after the $30 \mathrm{~m}$ muon shield at a distance approximately $90 \mathrm{~m}$ downstream from the target.

The production angle for pions (kaons) which decay into neutrinos above $1 \mathrm{GeV}$ in energy at the detector is $40(100) \mathrm{mrad}$. By comparison, the angular acceptance of the exit aperture of the dipole magnet from the center of the target is $105 \mathrm{mrad}$, and that of the midpoint of the decay tunnel from the center of the target is 35 mrad.

The predicted neutrino spectra for this configuration are shown in figs. 8 and 9. Fig. 8 gives the $\nu_{e}$ component of the flux through the detector from $\mathrm{K}_{\mathrm{e} 3}^{0}$ decays, while fig. 9 gives a breakdown of the different $\nu_{\mu}$ contributions according to parent secondary. Although the flux from pion decay has been suppressed by the sweeping magnet, it still remains the largest source of $\nu_{\mu}$ contamination in the $\nu_{\mathrm{e}}$ beam. We estimate the contamination of

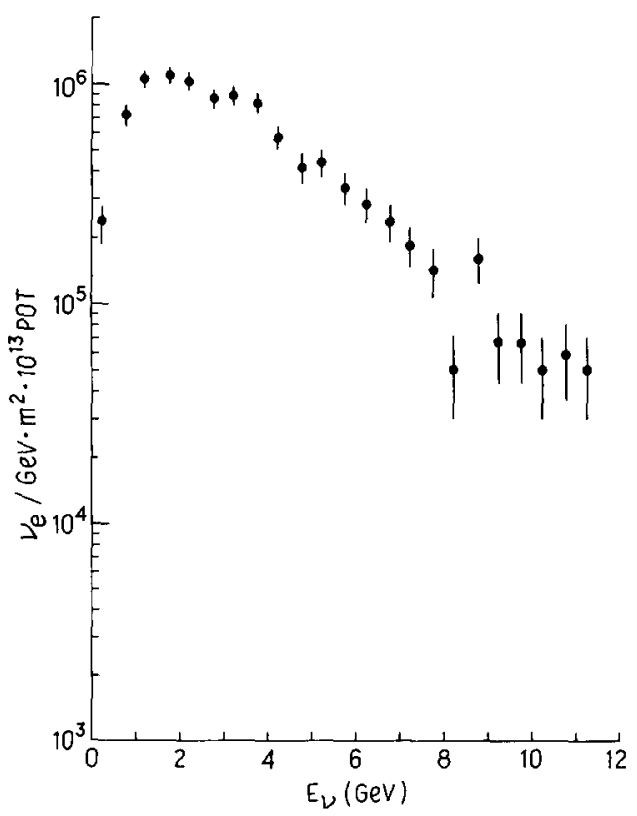

Fig. 8. Plot of predicted $\nu_{c}$ flux obtained using the physical parameters of table 3 and normalised to data of Murtagh et al.



Fig. 9. Plot of predicted $\nu_{\mu}$ flux obtained using parameters of table 3 and broken down into contributing components.

$\nu_{\mu}$ 's from $\Lambda^{0}$ decay to be less than $5 \%$ of that from pion and kaon decay. Integrating the curves in figs. 8 and 9 , we obtain a $\nu_{\mathrm{e}} / \nu_{\mu}$ ratio of $1 / 13.4$. One can take advantage of the $\nu_{\mathrm{e}}$ spectrum peak at $\sim 1 \mathrm{GeV}$ and the rapidly falling $\nu_{\mu}$ spectrum. An energy cut at $E_{\nu} \geq 1$ $\mathrm{GeV}$ improves the $\nu_{\mathrm{e}} / \nu_{\mu}$ ratio to $1 / 11.6$, while maintaining $90 \%$ of the $\nu_{\mathrm{e}}$ flux. Cutting at an energy of $1.5 \mathrm{GeV}$ gives only a marginal increase to $1 / 11.3$ decreasing the $\nu_{e}$ flux to $80 \%$ of its original value. The fluxes obtained with these energy cuts and the net $\nu_{\mathrm{e}} / \nu_{\mu}$ ratios are listed in table 7. Making the $1 \mathrm{GeV}$ energy cut yields a flux of $2.6 \times 10^{14} \nu_{\mathrm{e}}$ 's through the closest $3 \mathrm{~m}$ detector for $8.4 \times 10^{19}$ protons on target, a four month run.

Changing the field strength in the sweeping magnet and the length of the magnet strongly affects the $\nu_{e} / \nu_{\mu}$ ratio. This effect is shown in table 7 . Doubling the field

Table 7

$\nu_{c}$ fluxes and $\nu_{c} / \nu_{\mu}$ ratios for the enriched $\nu_{\mathrm{e}}$ beam

\begin{tabular}{llll}
\hline Magnetic field & Energy cut & $\begin{array}{l}\nu_{\mathrm{e}} \text { flux } \\
\left(\# / \mathrm{m}^{2} \mathrm{~s}\right)\end{array}$ & $\nu_{\mathrm{e}} / \nu_{\mu}$ ratio \\
\hline $22 \mathrm{kG}, 72 \mathrm{in}$. & none & $3.86 \times 10^{6}$ & $1 / 13.4$ \\
& $>1 \mathrm{GeV}$ & $3.47 \times 10^{6}$ & $1 / 11.6$ \\
& $>1.5 \mathrm{GeV}$ & $3.09 \times 10^{6}$ & $1 / 11.3$ \\
$44 \mathrm{kG}, 72 \mathrm{in.}$ & $>1 \mathrm{GeV}$ & $3.47 \times 10^{6}$ & $1 / 2.3$ \\
$22 \mathrm{kG}, 144 \mathrm{in.}$ & $>1 \mathrm{GeV}$ & $2.88 \times 10^{6}$ & $1 / 5.1$ \\
$44 \mathrm{kG}, 144 \mathrm{in}$. & $>1 \mathrm{GeV}$ & $2.88 \times 10^{6}$ & $1 / 2.0$ \\
\hline
\end{tabular}

a) Flux calculated in a 20 mrad detector at $90 \mathrm{~m}$ from the decay point. 
strength from 22 to $44 \mathrm{kG}$ increases the $\nu_{\mathrm{e}} / \nu_{\mu}$ ratio to $1 / 2.3$ for a neutrino energy above $1 \mathrm{GeV}$. Doubling the magnet length from six feet to twelve feet gives a $\nu_{\mathrm{e}} / \nu_{\mu}$ ratio of $1 / 5.1$. The $\nu_{e}$ flux is reduced to $83 \%$ of the flux with a six foot magnet due to the smaller effective aperture of the longer magnet. By doubling both the magnet field strength and length we obtain a $\nu_{\mathrm{e}} / \nu_{\mu}$ ratio of $1 / 2.0$ for neutrinos above $1 \mathrm{GeV}$ in energy.

\section{Discussion and conclusions}

Given the design parameters in table 3 for a $\nu_{\mathrm{e}}$ beam line, this design predicts $2.6 \times 10^{14} \nu_{\mathrm{e}}$ 's through a $3 \mathrm{~m}$ detector in four months, corresponding to a $\nu_{\mathrm{e}}$ flux of $3.5 \times 10^{6} \nu_{e} / \mathrm{m}^{2} \mathrm{~s}$. Recent results from proton decay experiments with detectors similar to the one proposed here indicate a typical $\nu_{\mu}: \nu_{\mathrm{e}}$ rejection ratio of $20: 1$ [21]. Adopting this rejection ratio as a guideline, the design parameters in table 3 predict a neutrino interaction signal to background of $\sim 2: 1$ in the detector. Reasonable event rates follow for a sufficiently large detector. The rates are significantly improved by the proposed increase in the BNL AGS proton yield from $2 \times 10^{13}$ protons per pulse to $1 \times 10^{14}$ protons per pulse [22]. With a marked increase in the primary proton yield we can also consider a longer and higher strength magnetic field while maintaining an acceptable event rate. Given a $44 \mathrm{kG}$ sweeping manget twelve feet long, and five times the proton flux from the AGS, we calculate $1.1 \times 10^{15} \nu_{\mathrm{e}}$ 's through the first detector module in four months. This rate corresponds to a $\nu_{\mathrm{e}}$ flux of $1.5 \times 10^{7}$ $\nu_{\mathrm{e}} / \mathrm{m}^{2} \mathrm{~s}$ with a $\nu_{\mathrm{e}}$ to $\nu_{\mu}$ ratio of $10: 1$ and a neutrino event rate of $700 / d$.

In conclusion, this study has shown that an experiment dedicated to the study of the time evolution of an electron neutrino beam is feasible using existing technology at a $30 \mathrm{GeV}$ proton machine. Good agreement has been obtained between this simulation and the $\nu_{\mu}$ spectra measured in the Brookhaven neutrino beam line. This agreement is, to our knowledge, a unique result.

We thank R. Bionta for his contributions to this work. One of the authors (R.A.O.) would like to acknowledge the use of SLAC computers in preparing this work, the help of $C$. Wendt in reading the manuscript, and the understanding of Prof. J. Jaros.

\section{References}

[1] H. Meyer et al. (CDHS Collaboration), Proc. Int. Europhysics Conf. on High Energy Physics '83, Brighton, England, eds., J. Guy and C. Costain, Rutherford Lab, Chilton England (1983) p. 393; W.H. Smith et al. (CCFR Collaboration), ibid., p. 395.

[2] F. Reines, H. Sobel and E. Pasierb, Phys. Rev. Lett. 45 (1980) 1307; Cavaignac et al., LAPP Exp-84-03 (May 1984).

[3] Bergsma et al. (CHARM Collaboration), CERN-EP/84-36 (1984).

[4] A. De Rujula et al., Nucl. Phys. B168 (1980) 54; V. Barger et al., J. Phys. G6 (1980) L165.

[5] Lubimov et al., Proc. HEP '83, p. 386; also Phys. Lett. 94B (1980) 266.

[6] T.B. Cleveland, R. Davis Jr. and J.K. Rowly, Proc. Neutrino Oscillations Workshop (1981) BNL 51380, p. 1.

[7] Recent inclusive $\nu_{\mu}$ measurements from the CDHS experiment at CERN over a range of $L / p$ from $0.3 \mathrm{~km} / \mathrm{GeV}$ to $0.04 \mathrm{~km} / \mathrm{GeV}$.

[8] H. Chen, Proc. of $\nu$ Oscillation Workshop (1981) p. 217.

[9] L.C. Teng, 1976 Summer Study on Kaon Factories, BNL 50579, p. 189.

[10] L. Sulak et al., Proc. of $\nu$ Oscillation Workshop (1981) p. 189.

[11] W. Lee et al., ibid., p. 171.

[12] The parameters presented in the text are a good representation of the extracted proton beam in the U-line of the AGS, just upstream of the present neutrino target. $G$. Smith, private communication.

[13] T. Eichten et al., Nucl. Phys. B44 (1972) 333.

[14] J.R. Sanford and C.L. Wang, Brookhaven Internal Report, JRS/CLW-1.2 (1967). A later report exists, but we chose the original parametrization since this version fits the angular distribution of the Eichten data better.

[15] L. Jauneau, Methods of Subnuclear Physics, vol. 3, ed., M. Nikolic (New York, 1969) p. 134.

[16] M. Murtagh, private communications.

[17] G. Smith, private communication.

[18] W. Lee et al., Proc. Int. Neutrino Conf., Aachen, Germany, ed., H. Faissner, H. Reithler and P. Zerwas (1976) p. 320.

[19] For example, C. Baltay and C.L. Wang, private communications. Also Blietschau et al., Nucl. Phys. B133 (1976) 205; Nucl. Phys. B144 (1976) 189 have fluxes calculated for the CERN wide band neutrino beam. These fluxes may be scaled to the Brookhaven parameters but the same reservations made in the text also apply.

[20] K. Raychaudhuri et al., Phys. Lett. 90B (1980) 319.

[21] M. Koshiba et al. (Kamioka Proton Decay Expt), Proc. 23rd Int. Conf. on High Energy Physics, Leipzig, DDR.

[22] B. Palmer, private communication. 